\title{
A new approach to assymmetric feedback in a segmented broad area diode laser
}

\author{
Jensen, Ole Bjarlin; Thestrup Nielsen, Birgitte; Petersen, Paul Michael
}

Published in:

Optics Communications

Link to article, DOI:

10.1016/j.optcom.2009.03.047

Publication date:

2009

Link back to DTU Orbit

Citation (APA):

Jensen, O. B., Thestrup Nielsen, B., \& Petersen, P. M. (2009). A new approach to assymmetric feedback in a segmented broad area diode laser. Optics Communications, 282(14), 2898-2900.

https://doi.org/10.1016/j.optcom.2009.03.047

\section{General rights}

Copyright and moral rights for the publications made accessible in the public portal are retained by the authors and/or other copyright owners and it is a condition of accessing publications that users recognise and abide by the legal requirements associated with these rights.

- Users may download and print one copy of any publication from the public portal for the purpose of private study or research.

- You may not further distribute the material or use it for any profit-making activity or commercial gain

- You may freely distribute the URL identifying the publication in the public portal

If you believe that this document breaches copyright please contact us providing details, and we will remove access to the work immediately and investigate your claim. 


\title{
A new approach to asymmetric feedback in a segmented broad
}

\section{area diode laser}

\author{
Ole Bjarlin Jensen, Birgitte Thestrup and Paul Michael Petersen \\ DTU Fotonik, Department of Photonics Engineering, Technical University of Denmark, P.O. Box 49, Dk-4000 \\ Roskilde, Denmark. \\ E-mail: ole.bjarlin.jensen@risoe.dk
}

\begin{abstract}
.
We present the demonstration of a non-critical setup for asymmetric feedback in a segmented broad area diode laser. We compare the dependence of the beam quality on the position of the dispersive element for standard spectral beam combining and our new non-critical setup. We find that our new approach is significantly less critical to the position of the dispersive element. It is shown that we can displace the dispersive element by at least $50 \%$ of the focal length of the collimating lens away from the Fourier plane without compromising performance. Furthermore, our approach provides the same high beam quality as is possible using off-axis spectral beam combining. This provides the possibility of achieving a high beam quality in a more compact setup than previously possible.
\end{abstract}

Keywords: Semiconductor laser, Beam quality, External cavity, Off-axis feedback. 


\section{Introduction}

In recent years there has been a remarkable advancement in the development of high power diode lasers and diode laser bars. However, the poor beam quality of standard broad area diode laser emitters imposes a limitation on the use of these lasers in many applications. Different approaches exist for achieving better beam quality from high power diode lasers including off-axis feedback [1-4] and injection locking to an external single-mode laser source [5,6]. The use of tapered diode lasers is an interesting approach for achieving Watt-level output powers in a near-diffraction limited beam $[7,8]$. In order to achieve higher output powers, diode laser bars consisting of several diode lasers placed next to each other are traditionally used. The beam quality of diode laser bars, however, is much worse than for the individual diode lasers in the bar and therefore it is a major challenge to improve the overall quality of the output beam. Spectral beam combining is a method to improve the beam quality for a diode laser bar to near the beam quality of the individual diode lasers. This improves the spatial brightness of the laser but reduces the spectral brightness. Spectral beam combining has previously been employed with a diode laser bar consisting of 11 broad area lasers [9] and with a segmented broad area diode laser consisting of two segments [10]. Off-axis feedback has also been employed directly to diode laser bars in order to decrease the beam divergence and thus improve the beam quality [11]. In this way, it has been possible to improve the beam quality by a factor of 5 . Recently, we have developed a new double-feedback off-axis spectral beam combining technique that improves the beam quality beyond the beam quality of the individual segments in the segmented laser [12]. The combination of spectral beam combining and off-axis feedback was also introduced by Jechow et al. [13] resulting in more than $10 \mathrm{~W}$ output power with $M^{2}<14$ from a broad area diode laser bar. Liu et al. [14] recently proposed to use coherent combination using off-axis feedback. This interesting approach simultaneously improves the spatial and spectral brightness of a diode laser bar. In standard spectral beam combining experiments, it is necessary to place the dispersive element very close to the Fourier plane in order to achieve a good beam quality. This makes the positioning of the components rather critical and the fixed distances in the setup limits the possibilities to decrease the size of the laser setup. In standard off-axis feedback to single emitter broad area diode lasers, the performance 
of the laser is also very dependent on the exact positioning of the feedback with respect to the Fourier plane.

In the present paper, we demonstrate a highly non-critical setup for asymmetric feedback to a segmented broad area laser. We show that, in contrast to standard spectral beam combining and standard off-axis feedback, it is possible to displace the diffraction grating far away from the Fourier plane and still achieve effective feedback with a large improvement in the overall beam quality of the laser. It is shown that we can displace the grating by at least $50 \%$ of the focal length of the collimating lens away from the Fourier plane and still obtain results similar to a setup with the grating placed in the Fourier plane.

\section{Experimental setup}

The diode laser is a 5-W diode laser (Coherent S-81-5000C-500) emitting at $808 \mathrm{~nm}$. The emitter width is $500 \mu \mathrm{m}$ and the emitter is split into five segments each with a width of approximately $90 \mu \mathrm{m}$ and separated by $10 \mu \mathrm{m}$. The laser front facet is standard coated with around $5 \%$ reflectivity and the back facet is high reflection coated. The free-running laser has a beam quality parameter $M^{2}=55$ at a drive current of 2.2 A corresponding to an output power of $785 \mathrm{~mW}$ whereas the beam quality of an individual segment is measured to $M^{2}=8.2$. A schematic of the experimental setup is shown in figure 1 under three different experimental conditions; A) with the dispersive element placed in the Fourier plane, B) and C) with the dispersive element placed out of the Fourier plane, beyond and before the Fourier plane, respectively.

The beam is collimated in the fast axis using an aspherical microlens mounted directly on the laser heat sink. The beam is collimated in the slow axis using an $80 \mathrm{~mm}$ focal length cylindrical lens. This lens provides a pseudo-far-field in the Fourier plane one focal length away from the lens, i.e. $80 \mathrm{~mm}$ behind the lens. In standard off-axis spectral beam combining, a diffraction grating is placed in the Fourier plane in order to obtain good spatial overlap of the beams from all segments on the grating. Here, however, we place the grating either before or behind the Fourier plane at the distance $d$ from the collimating lens resulting in a displacement $\Delta d$. The grating is a gold-coated ruled diffraction grating with 1200 grooves 
per $\mathrm{mm}$ and a blaze wavelength of $800 \mathrm{~nm}$. The first order diffraction efficiency of the grating is approximately $87 \%$ and the angle of incidence on the grating is approximately $21^{\circ}$. A plane output coupler with $18 \%$ reflectivity on the front side and uncoated on the back side is placed in the first order diffracted beam and aligned to obtain feedback to the laser. No attempts were made to optimize the output coupler reflectivity. A gold coated mirror with a sharp edge is placed in the beam path between the grating and the output coupler and a spatial filter is placed behind the mirror. In the far field of each laser segment, the beam exhibits the well-known twin-lobe intensity profile. The twin-lobe intensity profile consists of the different spatial modes of the broad area laser segment. By properly adjusting the mirror, it is possible to select a few of the spatial modes and selectively feed back one lobe of these modes to the laser segment. A few spatial modes are amplified and the remaining spatial modes are suppressed [15-17]. A spatial filter is inserted to further suppress the remaining spatial modes. The output from the laser system thus consists of selected amplified spatial modes from the different segments in the broad area laser.

\section{Experimental results}

Applying feedback to the laser lowers the laser threshold and also reduces the slope efficiency because of the losses induced by the double pass on the grating. The laser drive current is limited to $2.2 \mathrm{~A}$ due to a limited damage threshold of the grating used. At 2.2 A, the output power achieved in the configuration with the grating placed in the Fourier plane is $560 \mathrm{~mW}$. The laser threshold is $1 \mathrm{~A}$ and the slope efficiency is $0.51 \mathrm{~W} / \mathrm{A}$. This is to be compared to the free-running values of $1.55 \mathrm{~A}$ and $1.18 \mathrm{~W} / \mathrm{A}$ for the threshold and slope efficiency respectively. Moving the grating to positions before or behind the Fourier plane did not have any noticeable effect on the laser threshold and slope efficiency.

Moving the grating position immediately lowered the beam quality. However, by subsequently adjusting the gold coated mirror and output coupler it was possible to recover the good beam quality in the output beam from the laser. This is shown in figure 2 where the beam profile is shown for the laser with asymmetric feedback with three different grating displacements $\Delta d$ corresponding to situation $\mathrm{A}, \mathrm{B}$ and $\mathrm{C}$ 
in figure 1 at a drive current of $2.2 \mathrm{~A}$. Very similar beam profiles are obtained with the grating placed at different distances from the lens and a measurement of the beam quality parameter, $M^{2}$, reveals that the beam quality at the different lens-grating distances are almost identical. This is illustrated in figure $3 \mathrm{~A}$. A hyperbolic fit to the measured $1 / \mathrm{e}^{2}$ beam caustic was used to obtain $M^{2}$. This is in sharp contrast to ordinary spectral beam combining, where the grating must be placed within a very short distance from the Fourier plane to obtain a good beam quality as shown for our segmented broad area diode laser setup in figure 3B. The figures reveal that for ordinary spectral beam combining, the grating must be placed within approximately $1 \%$ of the focal distance of the slow axis collimating lens in order to obtain good beam quality. With our new approach, it is possible to displace the grating by at least $50 \%$ of the focal distance of the slow axis collimating lens with very little influence on the beam quality. We believe that the double feedback is responsible for this degree of freedom in the grating position. In classical spectral beam combining, the output coupler acts as both front and end mirror in the setup if the cavity is unfolded. In the present double feedback scheme, however, the gold coated mirror acts as the end mirror and the output coupler as front mirror if the cavity is unfolded. This introduces an extra degree of freedom in the setup compared to classical spectral beam combining.

We have also tried to change the position of the output coupler, gold-coated mirror and spatial filter. The setup is relatively insensitive to the axial position of these components as well. We have observed similar behavior for other segmented diode lasers.

When the grating is placed in the Fourier plane, the spectrum of the output beam shows a number of main peaks equal to the number of segments in the laser as expected for spectral beam combining $[9,12]$. This is not the case when the grating is moved away from the Fourier plane. The width of the spectrum stays approximately the same at $4 \mathrm{~nm}$, but the spectrum becomes more complicated with many peaks. This may be due to the fact that the spectral beam combination is not efficient with the grating placed out of the Fourier plane and the laser is operating in a new regime. An example of the emitted spectrum from the laser is given in figure 4 . 


\section{Conclusion}

We have demonstrated a new approach to asymmetric feedback in a segmented broad area diode laser. In contrast to standard spectral beam combining, we have shown that the position of the dispersive component can be chosen relatively freely without compromising the system performance. We obtain a

high beam quality of approximately $M^{2}=2.4$ using our approach even when the grating is displaced by $50 \%$ of the focal length of the collimating lens from the Fourier plane. This enables the realization of a compact laser system.

\section{Acknowledgements}

The authors wish to acknowledge the financial support from the Danish Research Agency under grants no. 26-04-0100 and 26-02-0020.

\section{References}

[1] M. Løbel, P.M. Petersen, P.M. Johansen, J. Opt. Soc. Am. B 15 (1998) 2000.

[2] V. Raab, R. Menzel, Opt. Lett. 27 (2002) 167.

[3] A. Jechow, V. Raab, R. Menzel, M. Cenkier, S. Stry, J. Sacher, Opt. Comm. 277 (2007) 161.

[4] B. Thestrup, M. Chi, B. Sass, P.M. Petersen, Appl. Phys. Lett. 82 (2003) 680.

[5] L. Goldberg, H.F. Taylor, J.F. Weller, D.R. Scifres, Appl. Phys. Lett. 46 (1985) 236.

[6] G.L. Abbas, S. Yang, V.W.S. Chan, J.G. Fujimoto, Opt. Lett. 12 (1987) 605.

[7] M.T. Kelemen , J. Weber, G. Kaufel, G. Bihlmann, R. Moritz, M. Mikulla, G. Weimann, Electron. Lett. 41 (2005) 1011.

[8] F. Dittmar, B. Sumpf, J. Fricke, G. Erbert, G. Traenkle, IEEE Phot. Tech. Lett. 18 (2006) 601.

[9] V. Daneu, A. Sanchez, T.Y. Fan, H.K. Choi, G.W. Turner, C.C. Cook, Opt. Lett. 25 (2000) 405.

[10] M. Chi, B. Thestrup, J.L. Mortensen, M.E. Nielsen, P.M. Petersen, J. Opt. A: Pure Appl. Opt. 5 (2003) S338.

[11] X. Gao, Y. Zheng, H. Kan, K. Shinoda, Opt. Lett. 29 (2004) 361. 
[12] O.B. Jensen, B. Thestrup, P.E. Andersen, P.M. Petersen, Appl. Phys. B 83 (2006) 225.

[13] A. Jechow, V. Raab, R. Menzel, Appl. Opt. 45 (2006) 3545.

[14] B. Liu, Y. Liu, Y. Braiman, Opt. Express 16 (2008) 20935.

[15] S. Wolff, A. Rodionov, V.E. Sherstobitov, H. Fouckhardt, IEEE Quantum Electron. 39 (2003), 448.

[16] B. Thestrup, M. Chi, P.M. Petersen, Proc. SPIE 5336 (2004) 38.

[17] M. Chi, N.S. Bøgh, B. Thestrup, P.M. Petersen, Appl. Phys. Lett. 85 (2004) 1107. 


\section{Figure captions}

Fig. 1. A schematic diagram of the experimental setup for asymmetric feedback to a segmented diode laser. The three described scenarios are illustrated with the grating placed in the Fourier plane (A), after the Fourier plane (B) and before the Fourier plane (C).

Fig. 2. Beam profile of the focused laser with asymmetric feedback and different grating displacements $\Delta d . \Delta d=-20 \mathrm{~mm}$ (thin line), $\Delta d=0 \mathrm{~mm}$ (bold line) and $\Delta d=25 \mathrm{~mm}$ (dashed line). The beam profiles have been offset for clarity. The drive current is $2.2 \mathrm{~A}$.

Fig. 3. Slow axis beam quality parameter $M^{2}$ measured versus grating displacement $\Delta d$ for asymmetric feedback (A) and ordinary spectral beam combining (B). The dotted line indicates the Fourier plane.

Fig. 4. Wavelength spectrum of the laser with asymmetric feedback at a current of $2.2 \mathrm{~A}$ and a grating displacement $\Delta d=-20 \mathrm{~mm}$. 
(A)

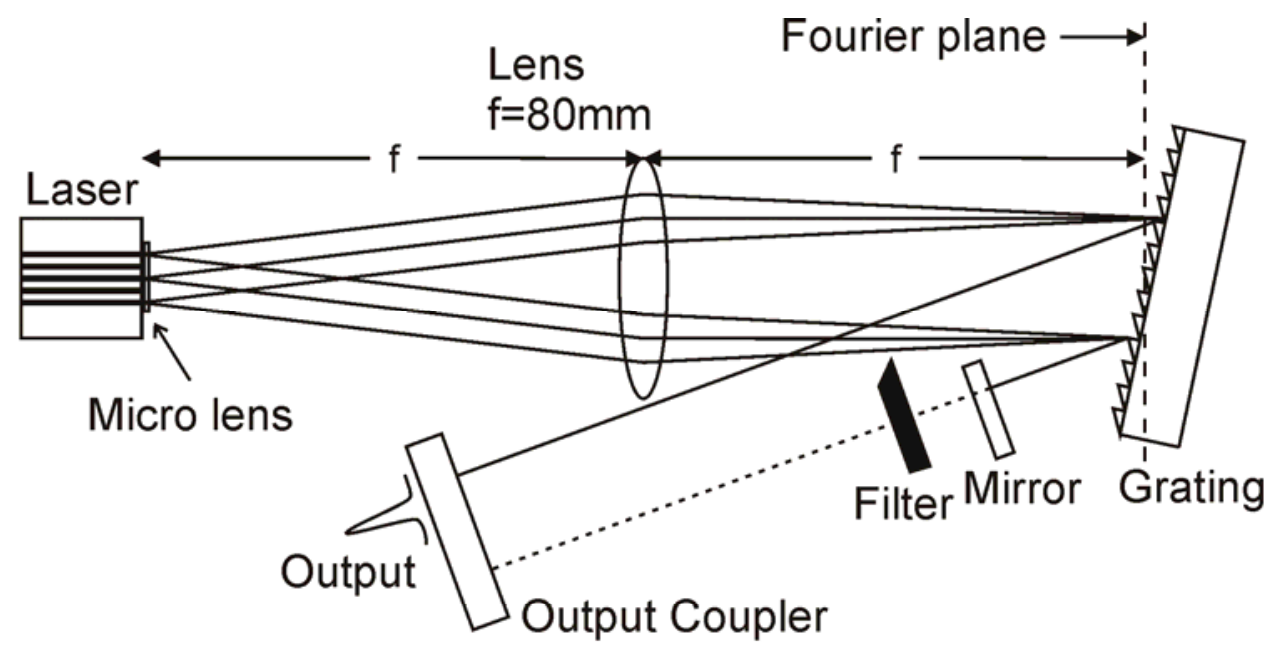

(B)

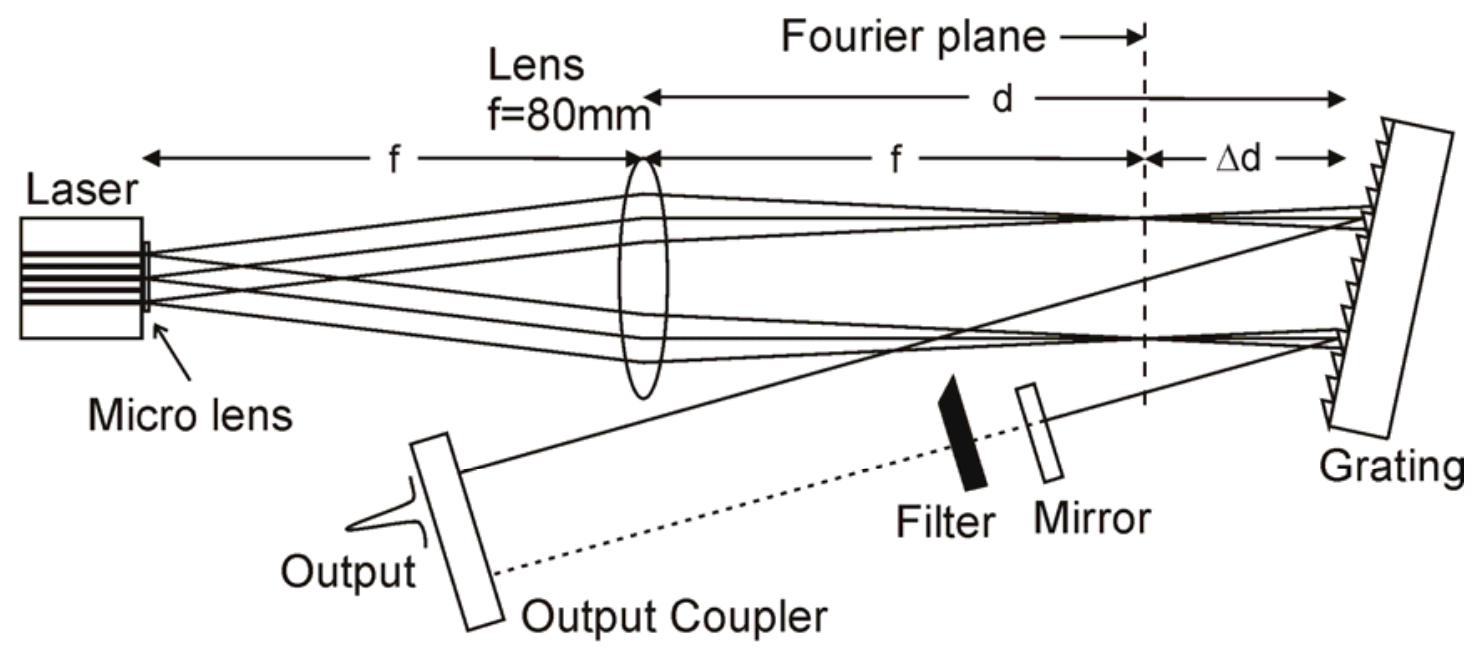

(C)

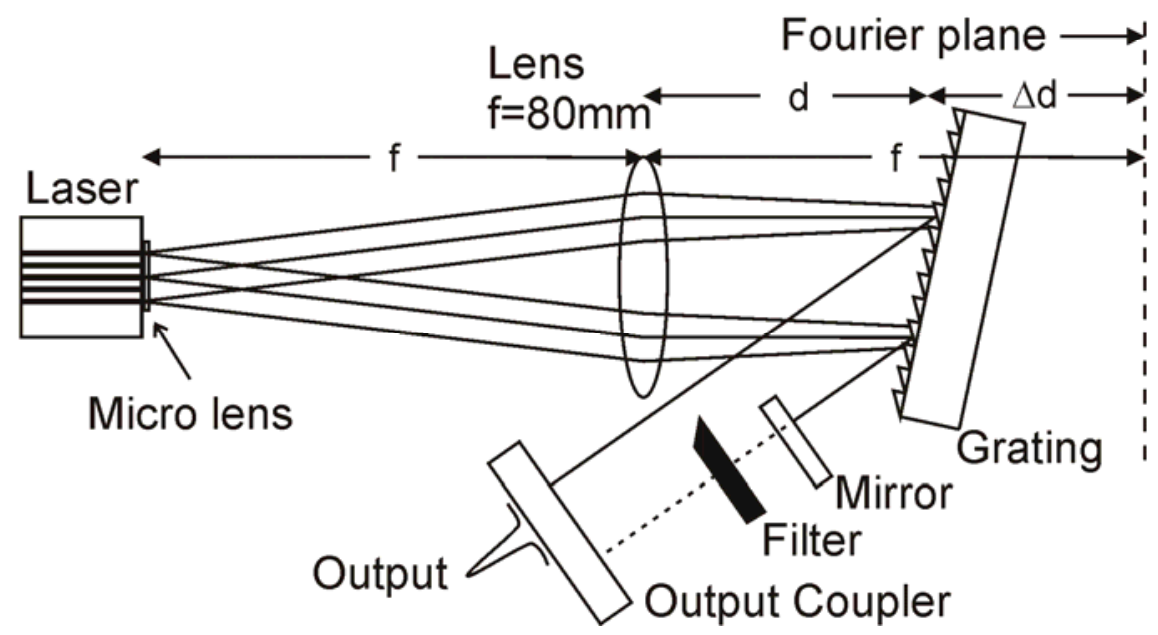

Figure 1. 


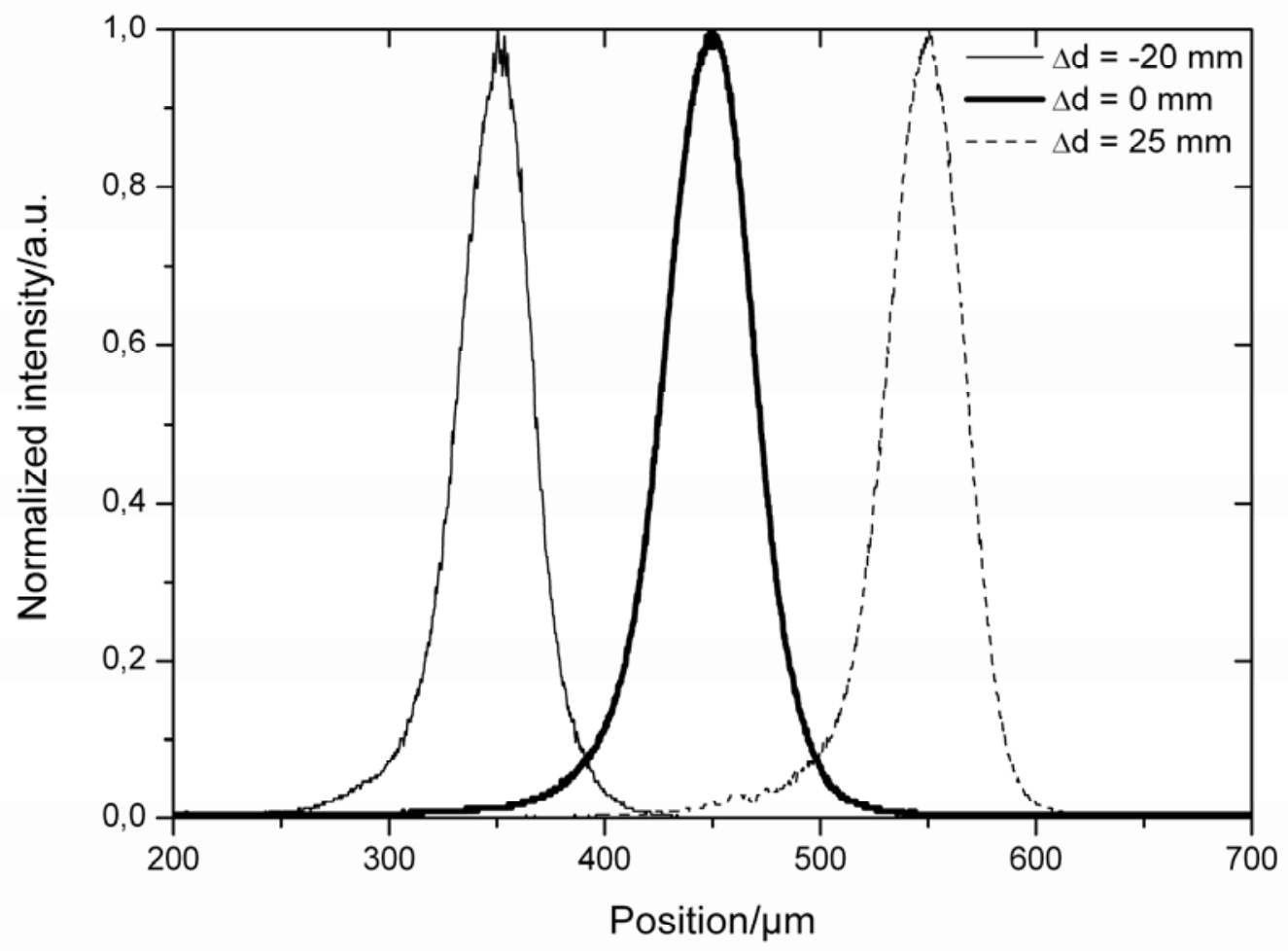

Figure 2. 


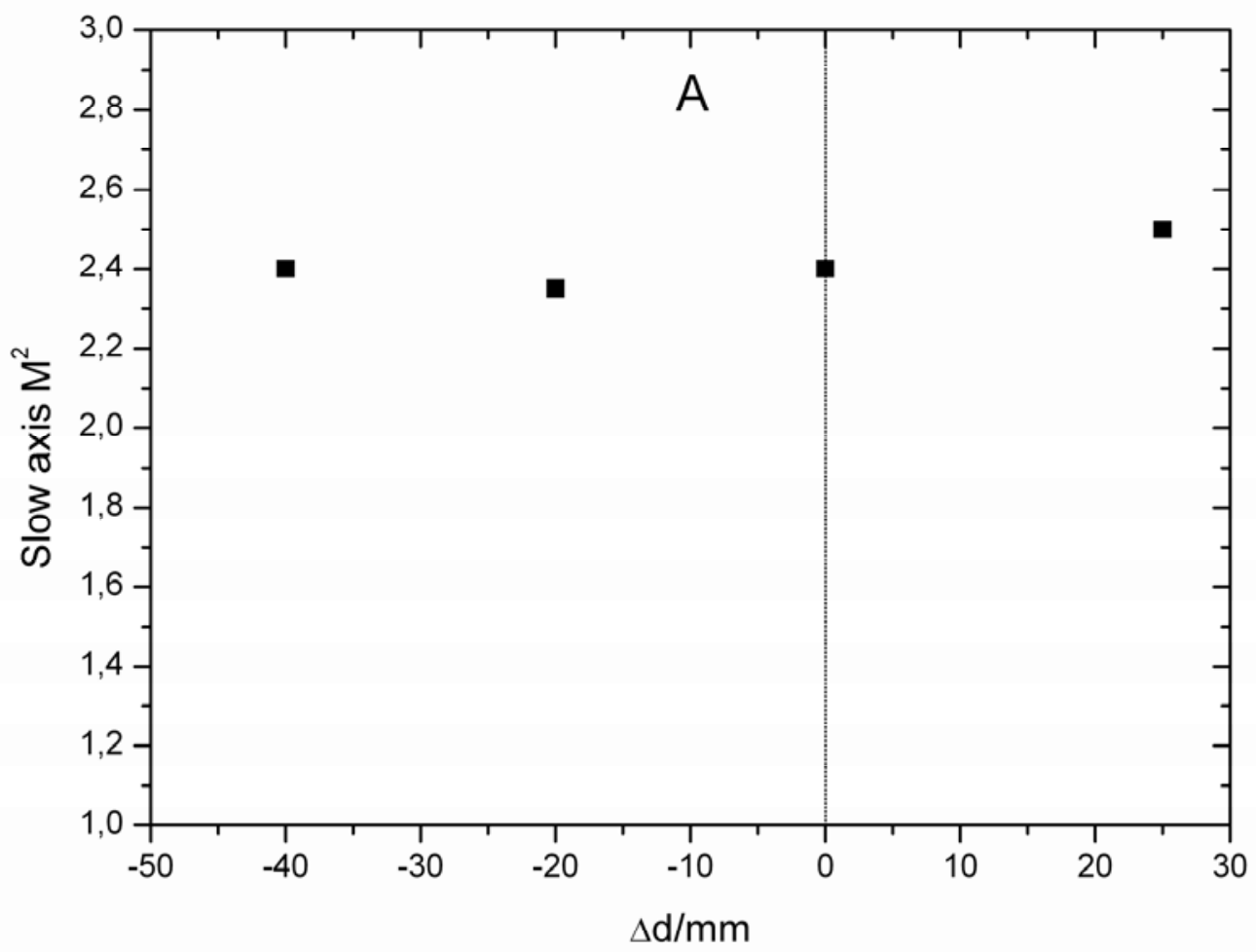

Figure 3A. 


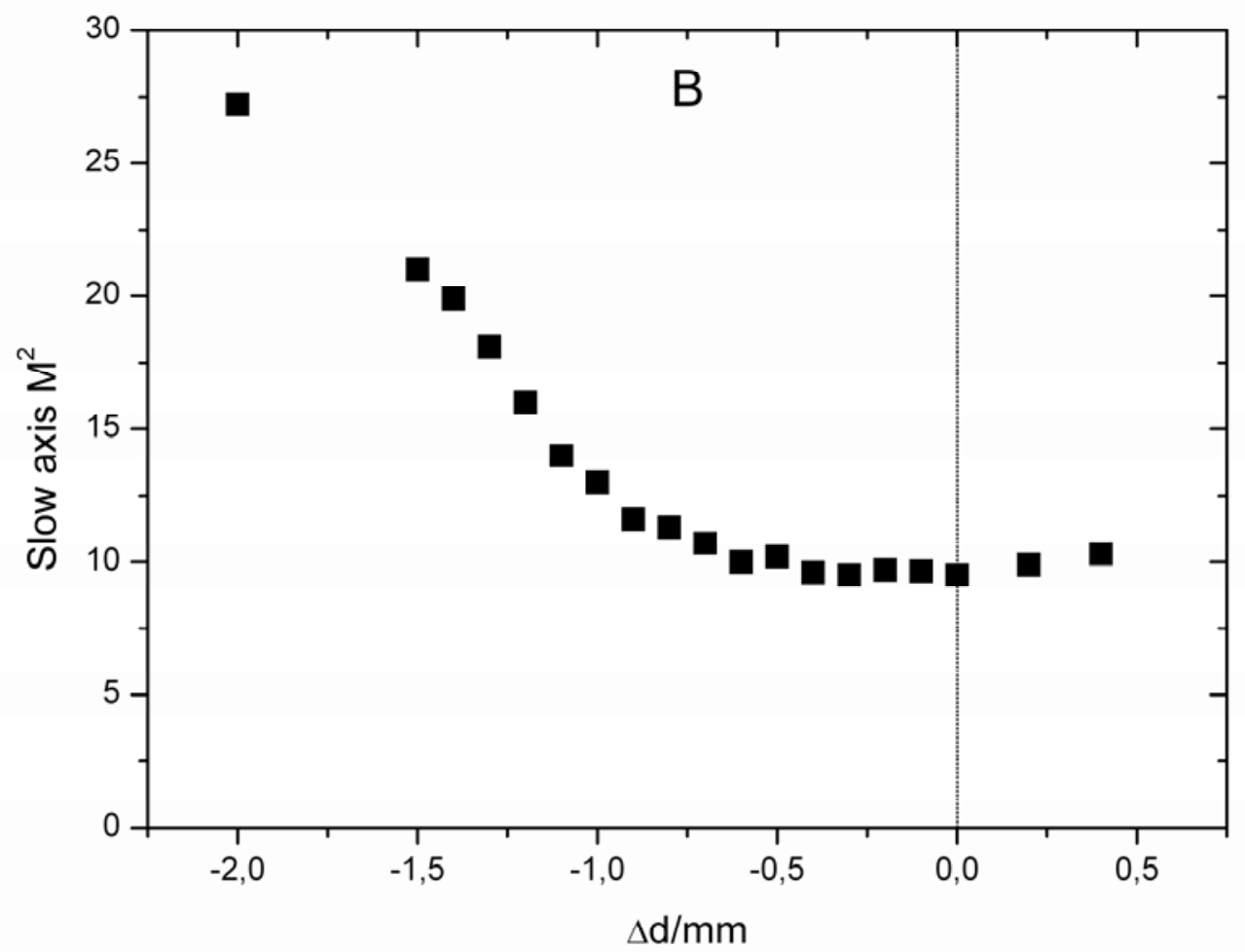

Figure 3B. 


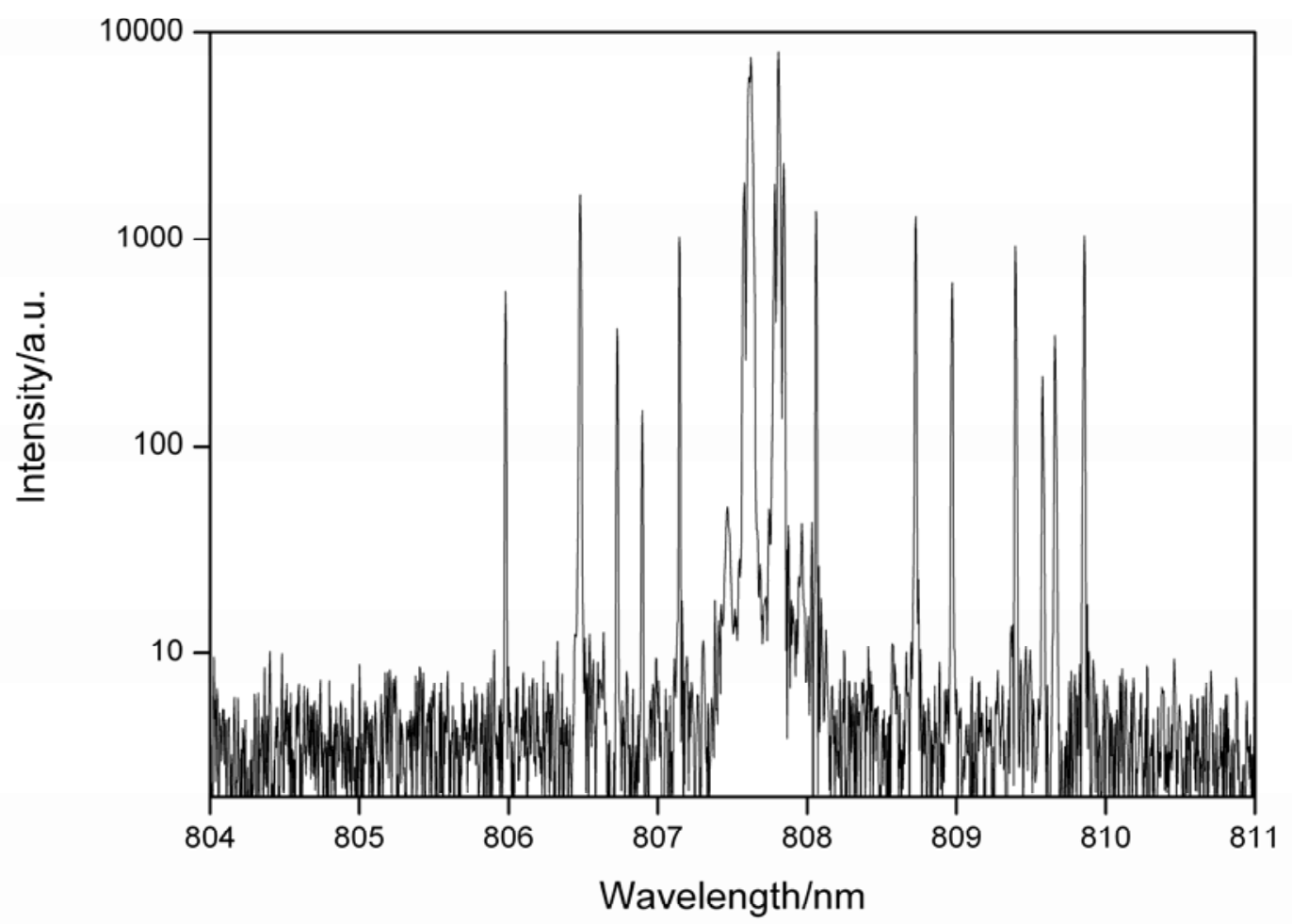

Figure 4. 